\title{
The effect of atrium façade design on daylighting in atrium and its adjoining spaces
}

\author{
S. Samant \\ Department of the Built Environment, University of Nottingham, UK
}

\begin{abstract}
Atrium buildings have been unsuccessful in utilizing available daylight in spaces adjoining the atria. Several studies indicate the potential to improve daylight in adjoining spaces through glazing distributions of progressive increase in the amount of openings from the upper to the lower floors in medium scale atria. The aim of this investigation was to study parametrically the effects of different glazing distributions on Daylight Factors (DFs) in the atrium and its adjoining spaces under overcast sky conditions. Using ECOTECT and RADIANCE, the objective was to provide optimum glazing ratios for a four sided, top-lit, square atrium building of WI 1.25. Three curves, each with five options of $20 \%, 30 \%$, $40 \%, 50 \%$ and $60 \%$ openings on top floor with a progressive increase in openings and $100 \%$ opening on the ground floor were tested. The study concluded that façade compositions have almost no influence on the lower floor adjoining spaces where more daylight is typically required, but can have significant influence on the top two floors. Having very small windows on the top floor $(20 \%)$ increases DFs in the adjoining spaces, however this increase is not significant and compromises light levels on the top two floors. In this study, the option of $60 \%$ opening on top floor, $79 \%$ opening on fourth floor, $92 \%$ opening on third floor, $98 \%$ opening on second floor and $100 \%$ opening on ground floor was recommended as it increased DFs on the top floors without compromising DFs on the lower floors.
\end{abstract}

Keywords: atrium, daylight, adjoining spaces, glazing ratios.

\section{Introduction}

The importance of daylight in atrium's environmental performance has led to several investigations of daylighting in atria and its adjoining spaces. Daylight 
levels within the atrium space are generally sufficiently high. However, this may not be the case for spaces adjoining the atria, where daylight varies significantly with every floor level. Rooms on the top floors can be over-lit and suffer from glare while daylight levels on the lower floors can be low, particularly in tall/deep atria.

Daylight performance (quantity and distribution) of an atrium and its adjoining spaces is complex and affected by the atrium's enclosing surfaces which determine how much light is going to be transmitted to the adjacent spaces, or reflected down towards the lower floors. This includes atrium facade design; its surface reflectances, window size/positioning, use of innovative daylighting systems (lightshelves, lightscoops); and atrium floor reflectances

\section{Literature review}

In an atrium well, Daylight Factor (DF) comprises of the sky component (SC) and the internally reflected component (IRC) from the atrium's enclosing surfaces (walls and floor). Therefore, wall reflectance has a direct and significant impact on inter-reflectance occurring inside the light well and determines the distribution of light in the space, and the amount of light which reaches the lower levels. CIBSE Code for Interior Lighting [1] recommended that reflectances of the atrium well facades should also be as high as possible to improve daylight in the adjoining space. However, the amount of increase would depend on the atrium form.

Several authors (Aschehoug [2]; Cole [3]; Boubekri [4]) suggest that the proportion of window area should vary between the floors of the atrium. Since most daylight is available at the top of the atrium, adjoining spaces need the smallest windows to achieve desired daylight levels. A progressive increase in the amount of openings from upper to the lower floors can lead to higher DFs available at the bottom of the atrium.

Aschehoug [2] studied daylight distribution in adjoining spaces with windows facing a glazed street of infinite length. This study presented an "optimum" glazing percentage for the facades facing a glazed space with $50 \%$ glazing on the $4^{\text {th }}$ floor, $60 \%$ glazing on the $3^{\text {rd }}$ floor, $70 \%$ glazing on the 2 nd floor and $100 \%$ glazing on the1st floor to give quite similar daylight conditions in rooms on all floors in the adjacent buildings.

Willbold-Lohr [5] studied different facade apertures in square shaped atria with a well index ranging from 0.5 to 2.0 . The study concluded that a facade aperture with $50 \%$ window openings will reduce the contribution of the IRC by half and having only glazed walls as separation between the office and the atrium the IRC is reduced to $1 / 3$ of the white walls, and almost reduced to the contribution of the skylight alone.

Cole [3] undertook scale model study to examine daylight factor distribution in the adjacent spaces of the ground floor, third and fifth floor respectively of an open, square, five storey atrium building for: $100 \%, 50 \%$ and variable openings into adjacent spaces. The study demonstrates that the variable opening option of $100 \%$ - Ground, $80 \%-2^{\text {nd }}, 60 \%-3^{\text {rd }}, 40 \%-4^{\text {th }}$ and $20 \%$ on $5^{\text {th }}$ floor - is the most 
effective in terms of bringing daylight on the lower floors of adjoining spaces in atrium buildings, where it is most needed".

Undertaking physical model studies for a linear atrium, Matusiak et al. [6] evidence that varying glazing area or glazing type result in a small but significant increase in daylight on the atrium floor, and improve balance of lighting in the adjoining spaces.

Sharples and Mahambrey [7] examined the effect of different distribution patterns of atrium wall reflectances (representing atrium wall surfaces in real buildings) on DF at various positions in the well of a square, four-sided, top-lit atrium under CIE overcast sky. The study concluded that atrium surfaces with wide bands of different reflectance values affect DFs at the base of the atrium. However, if these bands are narrow, DFs are not significantly altered by different reflectance distributions.

Calcagni and Paroncini [8] provided a relationship between the main architectural components of an atrium (geometry, material properties, fenestration system, atrium roof) and daylight conditions in the adjoining space and on the atrium floor. Eleven atrium (square and rectangular) cases, characterized by a different WI $(0.2$ to 1.47$)$, and atrium wall reflectance $(10 \%$, $30 \%, 50 \%, 70 \%, 90 \%$ ) were investigated under the CIE overcast sky. Simplified formulas (for atrium with and without roof) derived from Radiance were developed for preliminary prediction of horizontal DF on the atrium floor and in the adjacent rooms at a distance of 4 metres. The study concluded that for the atrium with no roof, when the WI increases from 0.2-0.75 DF values drop sharply, however when WI increases from $0.75-1.29$, quite similar DF values are achieved. This suggests that WI $>1.29$ would have limited influence on DF in spaces adjoining the atria.

Samant and Fang [9] made parametric changes to the distribution of reflectances of diffuse atrium well surfaces in atriums with a WI 0.5, 1.0 and 1.5. In agreement with Calcagni and Paroncini [8] it was concluded that the well reflectance distributions have limited influence on daylight distribution in shallow or wide medium sized atria but have practically no influence in tall atria.

\section{Methodology}

The aim of the experiments was to study parametrically the effects of different glazing distributions on DFs in the atrium and its adjoining spaces under overcast sky conditions. The objective was to provide optimum glazing ratios for a four sided, top-lit, square shaped, medium size atrium building.

The experiments were carried out using ECOTECT and RADIANCE building analysis software packages. No roof elements were used over the atrium well in order to reduce the number of variables under consideration. The lighting analysis tool within ECOTECT, under the "sky illumination model" option "CIE overcast sky" was chosen. The model simulated a square, five storeys, four sided top-lit atrium with full-scale dimensions of 16 metres length $\mathrm{x} 16$ metres width $\mathrm{x}$ 20 metres height corresponding to a WI of 1.25. The adjoining spaces had a floor to floor height of 4 metres, with the false ceiling of 1 metre at its underside for 
servicing, giving a clear height of 3 metres and a room depth of 9 meters. All dimensions were defined in terms of interior envelope dimensions. Reflectances of all surfaces were chosen to represent real buildings; atrium walls and floor were assigned $85 \%$ and $40 \%$ reflectance respectively whilst the adjoining space walls, floor and ceiling were assigned $60 \%, 40 \%$ and $95 \%$ reflectances respectively.

To establish the most effective distribution of windows, a pilot test was carried out to compare two window options using Aschehoug's optimum ratio of $50 \%$ glazing on the $4^{\text {th }}$ floor, $60 \%$ glazing on the $3^{\text {rd }}$ floor, $70 \%$ glazing on the $2^{\text {nd }}$ floor and $100 \%$ glazing on the 1 st floor with all the above experimental set up, for a four storey building:

-One continuous horizontal strip window with the top reveal at 3 metres in line with the underside of the false ceiling centred in plan

-Three vertical windows with the top reveal at 3 metres in line with the underside of the false ceiling centred in plan

The results of this comparison demonstrated that the three vertical windows provided better distribution of light in the adjoining spaces and therefore this option was chosen for the parametric modelling of atrium facades. Cut outs in the atrium facades were made to represent glazing positions, however no glazing was included.

The following three curve options as shown in Table 1 were developed to establish the optimum glazing ratios and to assess if a particular nature of progressive increase in glazing affected daylight performance. As shown in the table, each curve option includes five options with $100 \%$ openings on the first floor, followed by progressive increase in openings for the intermediate floors, and $20 \%, 30 \%, 40 \%, 50 \%, 60 \%$ openings on top floor. The three curves were developed on the basis of the following:

-Curve option 1 - A consistent and gradual increase in openings from top to bottom floors

-Curve option 2 - A shallow/slow increase in openings on the higher floors followed by a steep increase in openings on the lower floors

-Curve option 3 - A steep increase in openings on the higher floors followed by a shallow/slow increase in openings on the lower floors

It was decided to analyse the worst case scenario, i.e. north facade of the south floor plate on all levels. Measurement points for DF calculations represented a working plane height of 0.85 metres above the floor level. Horizontal DF measurements were taken for 5 positions on each floor: at the centre of the atrium, on the atrium wall, and $0.5 ; 3.2$ and 5.8 metres inside the adjoining space along its centre line and 6 metres on either sides of it, in line with the centre of the three window openings. On completion of data input in Ecotect, the models were exported to Radiance using the export manager tool for physical real lighting analysis. 
Table 1: $\quad$ Three curve options of glazing ratios (each with five glazing ratios) for parametric modelling.

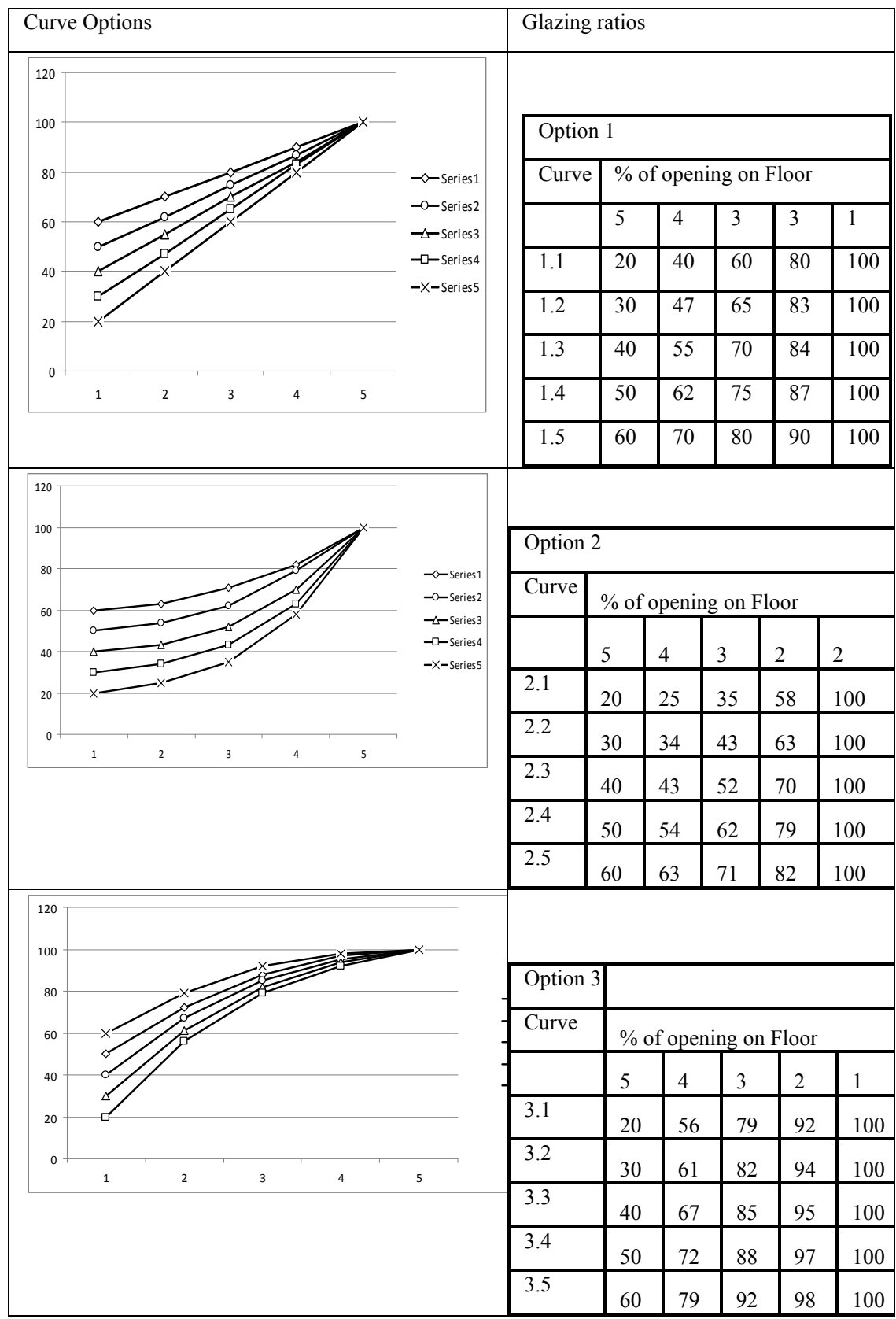




\section{Results and findings}

Variation in façade compositions had no influence on DFs at 5.8 metres into the adjoining space. On comparison of the three curve options, it is evident that there is a very good agreement between them. However, when results were compared with those obtained for curve option 1, curve option 3 gave marginally better DFs overall while curve option 2 gave slightly lower DFs for particular positions on third and fourth floor. This was mainly observed at $500 \mathrm{~mm}$ in the adjoining space when DFs for Curves 2.1, 2.2, 2.3 were 5\%-12\% lower and for Curves $3.1,3.2,3.3$ were $1 \%-15 \%$ higher than those obtained by Curves $1.1,1.2,1.3$ respectively.

For all the three curves, the options with $50 \%$ and $60 \%$ openings on the top floor (curves $1.4,1.5,2.4,2.5,3.4,3.5$ ) performed better than those with $20 \%$, $30 \%, 40 \%$ openings on the top floor (curves 1.1, 1.2, 1.3, 2.1, 2.2, 2.3, 3.1, 3.2, 3.3).

Comparing data for the curves 1.4, 1.5, 2.4, 2.5, 3.4, 3.5 in the four positions (atrium centre, atrium wall, at 0.5 and 3.2 metres in the adjoining space), DF values for the all three curves were very similar except at $500 \mathrm{~mm}$ into the adjoining space on the fourth and fifth floor when DFs for curves 1.4, 2.4, 3.4 were lower by $3 \%$ to $6.6 \%$ than those for $1.5,2.5,3.5$. Curves 1.5 and 3.5 gave very similar results and were the best curves, however given that curve 3 also performed best for 3.1, 3.2, 3.3 conditions, it was concluded that curve 3.5 with $60 \%$ opening on top floor, $79 \%$ opening on fourth floor, $92 \%$ opening on third floor, $98 \%$ opening on second floor and $100 \%$ opening on ground floor was the best option.

Whilst 1.4, 1.5, 2.4, 2.5, 3.4, 3.5 curves performed much better than the other options, these curves gave very similar DFs suggesting that the different progressive increases have limited influence on daylight quantity and its distribution in the adjoining spaces. Therefore, given the simplicity of curve 1 , its performance, probability and ease of use in practice, further investigation of Curve 1 was undertaken to understand its influence on daylight distribution in the atrium and its adjoining spaces on all floors.

Curves 1.1, 1.2, 1.3 provide quite similar DFs within the range of approximately $2.6 \%$ difference for the various points with the exception of the top two floors where DFs improved with increase in window sizes particularly at $500 \mathrm{~mm}$ inside the adjoining space. Notably, in comparison with 1.1 , DFs for 1.2 increased by $10 \%$ on the fourth floor at $500 \mathrm{~mm}$, and $2.3 \%$ on top floor at 3.2 metres inside the adjoining space. This trend continued for option 1.3 when compared to 1.2 resulting in $5 \%$ and $6.7 \%$ DF increase on the fourth floor and top floor at $500 \mathrm{~mm}$ inside the adjoining space respectively. Increase in openings on top floor from options 1.3 to 1.4 provided $3 \%$ increase on the fourth floor and $10.2 \%$ increase on top floor and for options 1.4 to 1.5 provided $6.6 \%$ increase on top floor at $500 \mathrm{~mm}$ inside the adjoining space. This demonstrates that the various options only affected DFs on the top two floors, mainly at $500 \mathrm{~mm}$ in the adjoining space, but did not improve DFs on the lower floors where more daylight is typically required. 
When comparing option 1.1 with $1.5, \mathrm{DF}$ at $500 \mathrm{~mm}$ inside the adjoining space for 1.5 improved significantly by $17.8 \%$ and $24.6 \%$ on fourth and fifth floor respectively. For $1.5, \mathrm{DF}$ at 3 metres inside the adjoining space also increased by $5.1 \%$ on the top floor and for all other points increase in DF ranged between $0.5 \%$ and $2.4 \%$. However, on the lower floors DFs were very similar for both the options and were in general higher by $0.1 \%$ and $2.6 \%$ for option 1.1 . This difference was mainly noted in the centre of the atrium position $(1.8 \%$ $2.6 \%)$ suggesting that the two options have very little influence $(0.1 \%-0.9 \%$ difference range in all other positions) on daylight availability in the lower adjoining floors. However, option 1.5 has significant influence on the top two floors and has the potential to affect daylight in these spaces.

Table 2: $\quad$ Difference in DFs for all positions for the five façade compositions $(1.1,1.2,1.3,1.4$, and 1.5).

\begin{tabular}{|c|c|c|c|c|c|}
\hline \multicolumn{6}{|c|}{ Maximum difference observed in DFs for the five options (1.1, 1.2, 1.3, 1.4, } \\
$1.5)$ \\
\hline $\begin{array}{c}\text { Floor } \\
\text { level }\end{array}$ & $\begin{array}{c}\text { Atrium } \\
\text { Centre }\end{array}$ & $\begin{array}{c}\text { Atrium } \\
\text { Wall }\end{array}$ & $\begin{array}{c}\text { At 0.5m in } \\
\text { Adjoining } \\
\text { Space }\end{array}$ & $\begin{array}{c}\text { At 3.2 m in } \\
\text { Adjoining } \\
\text { Space }\end{array}$ & $\begin{array}{c}\text { At 5.8m in } \\
\text { Adjoining } \\
\text { Space }\end{array}$ \\
\hline 1 & $1.8 \%$ & $1.3 \%$ & $0.7 \%$ & $0.3 \%$ & $0.1 \%$ \\
\hline 2 & $2.5 \%$ & $0.9 \%$ & $0.5 \%$ & $0.2 \%$ & $0.2 \%$ \\
\hline 3 & $2.6 \%$ & $0.8 \%$ & $1.3 \%$ & $0.1 \%$ & $0.2 \%$ \\
\hline 4 & $2.4 \%$ & $0.2 \%$ & $17.8 \%$ & $0.7 \%$ & $1.2 \%$ \\
\hline 5 & $0.8 \%$ & $1.1 \%$ & $24.6 \%$ & $5.1 \%$ & $1.4 \%$ \\
\hline
\end{tabular}

When the difference in DFs for all the positions on five floors for the five façade compositions were compared as shown in Table 2, it was observed that the variation in façade composition has limited influence on DFs $(0.8 \%-2.6 \%)$ at the centre of the atrium and on the atrium wall $(0.2 \%-1.3 \%)$. Variation in façade composition has very little influence on DFs in the adjoining spaces except for the top two floors and was most pronounced at $0.5 \mathrm{~m}$ in the adjoining space on all floors. Façade compositions have almost no influence on the lower floor adjoining spaces at 3 metres and beyond.

For all the five curves, DF at the atrium centre dropped by $65.9 \%-66.9 \%$ from top to bottom floor, and DF at the atrium wall dropped by $25.7 \%-28.1 \%$ from top to bottom floor. Whilst progressive increase in windows caused DFs at $500 \mathrm{~mm}$ in the adjoining space to increase by $9.3 \%, 8.4 \%$ and $1.2 \%$ from top to bottom floor for 1.1, 1.2 and 1.3 options respectively. However, for the same position fell by $6.6 \%$ and $15.6 \%$ from top to bottom floor for 1.4 and 1.5 options respectively.

For option 1.1, DFs from top to bottom floor increased by $1.0 \%$ at 3.2 metres, however for all the other options DFs from top to bottom floor decreased by 
$1.2 \%-4.3 \%$ at 3.2 metres and was more prominent for curves 1.5 and 1.4 where the DFs dropped by over 4\%. Whilst the drop in DFs on the lower floors for 1.4 and 1.5 might appear to be a negative effect, in reality it is only an increased difference between DF values between the top and bottom floors due to the increase in DFs on the top floors as a result of larger openings whilst maintaining DFs on the lower floors to those obtained by the other options.

It is evident that the horizontal DFs significantly vary in different positions higher up in the atrium but this difference gradually reduces from top to bottom floors. Additionally, there is a better agreement in DFs obtained from the five options $(1.1,1.2,1.3,1.4,1.5)$ lower down in the atrium than on the upper floors demonstrating that the effect of façade compositions is limited on the lower floors and distribution of light for all the five options on the lower three floors is similar.

Table 3: Shows the distribution of DF on each floor from centre to 3.2 metres in the adjoining space for the five options.

\begin{tabular}{|c|c|c|c|}
\hline Floor level & $\begin{array}{c}\text { Drop in DF from } \\
\text { Centre of atrium to } \\
\text { atrium wall \% }\end{array}$ & $\begin{array}{c}\text { Drop in DF from } \\
\text { Centre of atrium to } \\
0.5 \mathrm{~m} \text { in the } \\
\text { adjoining space } \%\end{array}$ & $\begin{array}{c}\text { Drop in DF from } \\
0.5 \mathrm{~m} \text { to } 3.2 \mathrm{~m} \text { in } \\
\text { the adjoining space } \\
\%\end{array}$ \\
\hline 1 & $4.3-5.5$ & $10.9-12.4$ & $13.1-13.6$ \\
\hline 2 & $11.0-12.7$ & $19.3-21.1$ & $17.0-17.4$ \\
\hline 3 & $18.5-20.6$ & $28.4-32.4$ & $20.5-21.9$ \\
\hline 4 & $30.8(1.5)-33.3(1.1)$ & $42.7(1.5)-63.0(1.1)$ & $8.8(1.1)-26.1(1.5)$ \\
\hline 5 & $43.7(1.5)-45.7(1.1)$ & $62.2(1.5)-87.7(1.1)$ & $5.2(1.1)-24.8(1.5)$ \\
\hline
\end{tabular}

For all the five options, on level 1 the drop in DF ranges between approximately $4 \%-5 \%$ from centre to atrium wall position, $11 \%-12 \%$ from centre to $0.5 \mathrm{~m}$ in the adjoining space and approximately $13 \%$ from 0.5 to 3.2 metres in the adjoining space (Table 3). On level 2, DF drops by $11 \%-13 \%$ from centre to atrium wall position, $19 \%-21 \%$ from centre to $0.5 \mathrm{~m}$ in the adjoining space and approximately $17 \%$ from 0.5 to 3.2 metres in the adjoining space. On level 3, DF drops by $18 \%-20 \%$ from centre to atrium wall position, $28 \%-32 \%$ from centre to $0.5 \mathrm{~m}$ in the adjoining space and approximately $20 \%$ to $22 \%$ from 0.5 to 3.2 metres in the adjoining space. On level 4 and 5, DF drops by $31 \%-33 \%$ and $44 \%-46 \%$ from centre to atrium wall position; $43 \%-63 \%$ and $62 \%-88 \%$ from centre to $0.5 \mathrm{~m}$ in the adjoining space, and approximately $9 \%$ to $26 \%$ and $5 \%-25 \%$ from 0.5 to 3.2 metres in the adjoining space respectively. There is a very big drop in DFs from centre to $0.5 \mathrm{~m}$ in the adjoining space on level 4 and 5 for option 1.1 due to very small windows on this floor but the DF drop reduces further into the adjoining space due to reduction in available light at 3.2 metres in the space. Whilst there is a bigger drop for option 1.5 from $0.5 \mathrm{~m}$ to 
3.1 metres into the adjoining space on the top two floors, the overall light levels are much higher.

\section{Conclusions}

The study concluded that for a medium sized building progressive increase in glazing from top to bottom floor has limited influence on daylight in the atrium space and its adjoining spaces allowing flexibility in façade design, whilst maintaining the design integrity and general trend in the ratios.

Variation in façade compositions has limited influence on DFs $(0.8 \%-2.6 \%)$ at the centre of the atrium and on the atrium wall $(0.2 \%-1.1 \%)$. Façade compositions have almost no influence on the lower floor adjoining spaces where more daylight is typically required, but can have significant influence on the top two floors.

Having smaller windows on the top floor $(20 \%)$ increases DFs in the adjoining spaces; however this increase is not significant and compromises DFs on the top two floors. However, a more gradual increase in windows with $50 \%$, $60 \%$ openings on top floor, increasing up to $100 \%$ on the lowest floor increases DFs on the top floors without significantly compromising DFs on the lower floors.

When comparing the $20 \%$ with the $60 \%$ option of windows on top floor, DF at $0.5 \mathrm{~m}$ inside the adjoining space improved significantly by $17.8 \%$ and $24.6 \%$ on $4^{\text {th }}$ and $5^{\text {th }}$ floor respectively. DF at 3 metres inside the adjoining space also increased by $5 \%$ on the top floor and for all other points increase in DF ranged between $0.5 \%$ and $2.4 \%$.

In this study, for a five storey building with a WI of 1.25 , curve option 3 with $60 \%$ opening on top floor, $79 \%$ opening on fourth floor, $92 \%$ opening on third floor, $98 \%$ opening on second floor and $100 \%$ opening on ground floor performed the best.

Whilst the drop in DFs on the lower floors for the $50 \%$ and $60 \%$ options might appear to be a negative effect, in reality it is only an increased difference between DF values between the top and bottom floors due to the increase in DFs on the top floors as a result of larger openings whilst maintaining DFs on the lower floors to those obtained by the other options $(20 \%, 30 \%, 40 \%)$.

At 5.8 metres inside the adjoining space, DFs were nearly the same for all the options but varied slightly for 3.2 metres and more significantly at 0.5 metres into the space, suggesting that glazing distributions do not affect DFs beyond 3 metres into the adjoining space. Horizontal DFs significantly reduce from centre to the atrium wall and drop further into the adjoining space. This effect is more noticeable on the upper floors; however the rate of decay gradually reduces from top to bottom floors.

This study could be extended to different atrium types -three sided, linear, and stepped atria to assess the performance of the chosen glazing options in different geometries. Additionally, further experiments could be conducted to include different types of wall fenestrations, light directing elements such as light- 
shelves and light-scoops, and cladding materials to assess their impact on available light in an atrium and its adjoining spaces.

\section{Acknowledgements}

The author would like to thank Mrs Sheetal Merai for her contribution in parametric modelling of this research project. Sheetal completed her Masters in Architecture, at the University of Nottingham in 2008 and currently works in a private architectural practice in Leeds.

\section{References}

[1] The Chartered Institution of Building Services Engineers, (1984). CIBSE CODE FOR INTERIOR LIGHTING, London.

[2] Aschehoug, O. (1986). Daylight Design for Glazed Spaces. PROCEEDINGS FROM THE INTERNATIONAL DAYLIGHTING CONFERENCE 2, Long Beach, CA, November, 237-243.

[3] Cole, R.J., (1990). The Effect of the Surfaces Enclosing Atria on the Daylight in Adjacent Spaces. BUILDING AND ENVIRONMENT, 25(1), 37-42.

[4] Boubekri, M. (1995). The Effect of the Cover and Reflective Properties of a Four Sided Atrium on the Behaviour of Light. ARCHITECTURAL SCIENCE REVIEW, 38(3), 3-8

[5] Willbold-Lohr, G. (1989) Daylighting in Glazed Atria. PROCEEDINGS OF THE 2ND EUROPEAN CONFERENCE ON ARCHITECTURE, Paris, 16-20.

[6] Matusiak, B., Aschehoug, O., Littlefair, P. (1999). Daylighting Strategies for an Infinitely Long Atrium: An Experimental Evaluation. LIGHTING RESEARCH AND TECHNOLOGY, 31(1), 23-34

[7] Sharples, S. and Mahambrey, S. (1999). Reflectance Distributions and Atrium Daylight Levels: A Model Study LIGHTING RESEARCH \& TECHNOLOGY, 31(4), 165-170

[8] Calcagni, B. and Paroncini, M. (2004). Daylight Factor Prediction in Atria Building Designs. SOLAR ENERGY, 76 (6) 669-682

[9] Samant, S. and Yang, F., (2007). Daylighting in Atrium: The Effect of Atrium Geometry and Reflectance Distributions. INTERNATIONAL JOURNAL OF LIGHTING RESEARCH AND TECHNOLOGY, 39(2), 147158 